\title{
Is rs34861192 or rs1862513 a more promising variant for determining plasma resistin in an aged Japanese population?
}

\author{
H. Osawa • Y. Tabara • J. Ohashi • R. Kawamura • \\ H. Onuma $\cdot$ H. Makino
}

Received: 21 September 2009 / Accepted: 7 December 2009/Published online: 4 February 2010

(C) The Author(s) 2010. This article is published with open access at Springerlink.com

Keywords Gene expression · Insulin resistance · Plasma . Polymorphism $\cdot$ Protein $\cdot$ Resistin $\cdot$ Serum $\cdot$ SNP

\section{Abbreviations \\ LD Linkage disequilibrium \\ SNP Single nucleotide polymorphism}

To the Editor: In a recent paper, Asano et al. reported on an analysis of single nucleotide polymorphisms (SNP) of the human resistin gene (RETN) in an aged Japanese popula-

H. Osawa $(\bowtie) \cdot$ R. Kawamura $\cdot$ H. Onuma $\cdot$ H. Makino

Department of Molecular and Genetic Medicine,

Ehime University Graduate School of Medicine,

Shitsukawa,

Toon, Ehime 791-0295, Japan

e-mail: harosawa@m.ehime-u.ac.jp

\section{Y. Tabara}

Department of Basic Medical Research and Education, Ehime University Graduate School of Medicine,

Toon, Ehime, Japan

\section{J. Ohashi}

Doctoral Program in Life System Medical Sciences, Graduate School of Comprehensive Human Sciences, University of Tsukuba,

Tsukuba, Ibaraki, Japan

H. Osawa $\cdot$ Y. Tabara $\cdot$ H. Onuma

Ehime Proteo-Medicine Research Center, Ehime University,

Toon, Ehime, Japan

\section{H. Makino}

Institute of Diabetes Research Center, Takanoko Hospital, Matsuyama, Ehime, Japan tion, and suggested that rs34861192 (SNP-638G>A) and rs3745368 (3' UTR) are significant determinants of plasma resistin [1]. We wish to report that this statement may cause readers to conclude erroneously that rs34861192, but not rs 1862513 (SNP-420C $>$ G), is a causal variant determining circulating resistin. If the functional data were to be taken into account, the interpretation of the genetic data would be different.

It appears to us that rs1862513 can still be considered to be a promising candidate for a causal variant, as evidenced from findings available to date, including their reported results [1-5]. First, it should be noted that their conclusion was drawn entirely from statistical analyses of genetic data. These data should be interpreted with caution, since it is not possible to assess the effect of A of rs34861192 independent of $\mathrm{G}$ of rs1862513, due to the absence of individuals having both the A/A genotype of rs34861192 and the $\mathrm{C} / \mathrm{C}$ genotype of rs1862513. Second, their conclusion was not verified by functional analyses. Of rs34861192, rs1862513 and rs3219175 (SNP-358G $>$ A, which is in complete linkage disequilibrium [LD] with rs34861192), functional roles have been proven only for rs1862513 in vitro [2-5].

Most crucially, when the data in Fig. 3 of Asano et al. [1] are inspected carefully, all individuals with the $\mathrm{A} / \mathrm{A}$ genotype of rs34861192 also had the G/G genotype of rs1862513. There were no individuals with both the A/A genotype of rs34861192 and the C/C genotype of rs1862513. Therefore, it is not possible to assess the effect of A of rs34861192 independent of $G$ of rs1862513. Individuals with the A/A genotype of rs34861192 and the $\mathrm{C} / \mathrm{C}$ genotype of rs1862513 should have been included in the analysis in order to determine which SNP is more critical for determining circulating resistin levels. This does not appear to be feasible due to the very low frequency of 
individuals with the combination of these two genotypes, which may be due to limitations in genetic epidemiology because of the available sample size. In addition, rs34861192, rs1862513 and rs3219175 are all located in the same LD block, and all of these three SNPs are strongly associated with circulating resistin levels. Based on their data, rs34861192 and rs1862513 are in moderate LD $\left(r^{2}=\right.$ 0.47). When calculated from our previous data in 200 type 2 diabetic cases and in 200 controls [2], $r^{2}$ was similar to that of Asano et al. $\left(r^{2}=0.51\right.$ and 0.57 , respectively), whereas $D^{\prime}$ was 1 in both groups. It should be pointed out that it is difficult to identify a causal variant from highly correlated SNPs by the exclusive use of statistical analyses of genetic data, in the absence of other information [6].

From the functional point of view, four previously published papers, including ours, have shown that RETN promoter activity with $\mathrm{G}$ of rs 1862513 is higher than that with $\mathrm{C}$ (see Table 1 for summary) [2-5]. Of these papers, two suggested that the effect of $G$ of rs 1862513 is independent of A of rs34861192. Using SL2 cells, we previously showed that $\mathrm{Sp} 1$ and $\mathrm{Sp} 3$ transcription factors enhance RETN promoter activity with G of rs1862513 in the absence of rs34861192 [2]. Smith et al. showed that promoter activity with $\mathrm{G}$ of $\mathrm{rs} 1862513$ is $\sim 300 \%$ higher than that with $\mathrm{C}$ of rs1862513 in 3T3-L1 adipocytes, without rs34861192 [5]. In addition, we reported that Sp1 and Sp3 transcription factors specifically bind to DNA sequences with $\mathrm{G}$ of rs 1862513 [2]. In contrast, Azuma et al. reported that the combination of risk alleles, A of rs34861192 and $\mathrm{G}$ of rs1862513, showed a higher RETN promoter activity than the other combinations of these SNPs, suggesting that A of rs 34861192 and G of rs 1862513 may both be required for the highest activity. There have been no reports on the clear function of rs34861192 and rs3219175 to date.
It should be noted that one cannot conclude that rs 1862513 is more promising than rs34861192. First, the association of plasma resistin with rs34861192 is much stronger than that for $\mathrm{rs} 1862513\left(1 \times 10^{-60}\right.$ vs $1 \times 10^{-24}$, respectively) [1]. Given the moderate correlation between the two SNPs $\left(r^{2}=0.47\right)$, it appears likely that this association signal is driven by rs34861192. The effect of rs1862513 appears to become more evident in the presence of rs34861192, although functional data in support of this is not available at this point. Second, the association between rs1862513 and circulating resistin had been reported in Japanese and Korean individuals [1, 2, 4], but not in white individuals $[7,8]$. Since rs34861192/rs3219175 are nearly monophormic (very low frequencies of risk alleles) in white individuals (dbSNP [www.ncbi.nlm.nih.gov/snp] and dbHAPMAP [www.hapmap.org/index.html.en]), the risk alleles of these SNPs, together with that of rs1862513, may both be required for the highest circulating resistin levels.

In summary, it should not be simply concluded that rs34861192 is a stronger candidate for determining plasma resistin concentration than rs1862513, based on the exclusive use of statistical analyses of their genetic data [1]. From the genetic point of view, it is difficult to determine which is the most promising candidate, since rs34861192, rs1862513, and rs3219175 are in the same LD block and are strongly correlated with plasma resistin, where the sample size used was limited. From the functional data available to date, a significant function has been proven only for rs 1862513 [2-5]. Therefore, at this point, it appears that rs 1862513 can still be considered to be a promising candidate for a causal variant, with the possibility that a certain combination of these three SNPs, including $\mathrm{G}$ of rs1862513, may have the strongest activity. The function of these SNPs, including interactions between

Table 1 Comparison of human resistin gene promoter activities between $\mathrm{G}$ and $\mathrm{C}$ of rs 1862513 in previous papers

\begin{tabular}{llllll}
\hline $\begin{array}{l}\text { Authors } \\
\text { [reference no.] }\end{array}$ & $\begin{array}{l}\text { rs34861192 } \\
(-638 \mathrm{G}>\mathrm{A})\end{array}$ & $\begin{array}{l}\text { rs1862513 } \\
(-420 \mathrm{C}>\mathrm{G})\end{array}$ & $\begin{array}{l}\text { rs3219175 } \\
(-358 \mathrm{G}>\mathrm{A})\end{array}$ & $\begin{array}{l}\text { Promoter activity } \\
\text { (fold induction) }\end{array}$ & Cell type \\
\hline Osawa et al. [2] & - & $\mathrm{G}$ & $\mathrm{G}$ & $\times 4$ (Sp1), ×10(Sp3) & SL2 \\
Azuma et al. [3] & $\mathrm{A}$ & $\mathrm{G}$ & $\mathrm{G}$ & $\times 1.2$ & THP1, 3T3-L1 (fibroblast) \\
Cho et al. [4] & $\mathrm{N}$ & $\mathrm{G}$ & $\mathrm{N}$ & $\times 1.4$ & 3T3-L1 (fibroblast) \\
Smith et al. [5] & - & $\mathrm{G}$ & $\mathrm{N}$ & $\times 4$ & 373-L1 (adipocyte) \\
\hline
\end{tabular}

Fold induction of promoter activity with $\mathrm{G}$ of rs1862513 is compared with that with $\mathrm{C}$ of rs1862513, whereas the other SNPs are unchanged. A of rs34861192, G of rs 1862513 and A of rs3219175 are risk alleles for higher serum resistin in humans. It should be noted that Azuma et al. [3] compared promoter activity with A of rs34861192 and G of rs 1862513 (both risk alleles) with that with G of rs 34861192 and $\mathrm{C}$ of rs 1862513 (both non-risk alleles), but stated that the other two promoter reporters with risk/non-risk alleles combination had activities similar to that with the both non-risk alleles. In all their reporter constructs, rs3219175 was G

$\mathrm{N}$, a nucleotide of the SNP included in the promoter reporter constructs is not specified; -, the SNP is not included in the promoter reporter constructs 
them, remains to be elucidated. Further experiments will be required to clarify these points.

Duality of interest The authors declare that there is no duality of interest associated with this manuscript.

Open Access This article is distributed under the terms of the Creative Commons Attribution Noncommercial License which permits any noncommercial use, distribution, and reproduction in any medium, provided the original author(s) and source are credited.

\section{References}

1. Asano H, Izawa H, Nagata $\mathrm{K}$, et al. (2010) Plasma resistin concentration determined by common variants in the resistin gene and associated with metabolic traits in an aged Japanese population. Diabetologia. doi:10.1007/s00125-009-1517-2

2. Osawa H, Yamada K, Onuma H et al (2004) The G/G genotype of a resistin single-nucleotide polymorphism at -420 increases type 2 diabetes mellitus susceptibility by inducing promoter activity through specific binding of Sp1/3. Am J Hum Genet 75:678-686

3. Azuma K, Oguchi S, Matsubara Y et al (2004) Novel resistin promoter polymorphisms: association with serum resistin level in Japanese obese individuals. Horm Metab Res 36:564-570

4. Cho Y, Youn B, Chung S et al (2004) Common genetic polymorphisms in the promoter of resistin gene are major determinants of plasma resistin concentrations in humans. Diabetologia 47:559565

5. Smith S, Bai F, Charbonneau C, Janderova L, Argyropoulos G (2003) A promoter genotype and oxidative stress potentially link resistin to human insulin resistance. Diabetes 52:1611-1618

6. Ioannidis JP, Thomas G, Daly MJ (2009) Validating, augmenting and refining genome-wide association signals. Nat Rev Genet $10: 318-329$

7. Hivert MF, Manning AK, McAteer JB et al (2009) Association of variants in RETN with plasma resistin levels and diabetesrelated traits in the Framingham Offspring Study. Diabetes $58: 750-756$

8. Menzaghi C, Coco A, Salvemini L et al (2006) Heritability of serum resistin and its genetic correlation with insulin resistancerelated features in nondiabetic Caucasians. J Clin Endocrinol Metab 91:2792-2795 